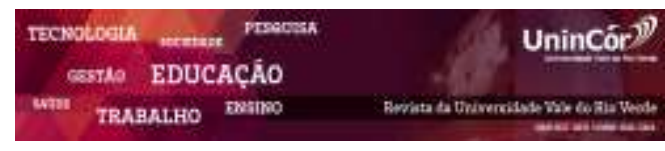

Revista da Universidade Vale do Rio Verde ISSN: 1517-0276 / EISSN: 2236-5362 Vol. 16 | n. 1 | Ano 2018

Reginaldo da Silva Souza

Universidade Vale do Rio Verde

Pontifícia Universidade Católica de Minas Gerais reginaldo-vga@ hotmail.com

Sheldon William Silva

Centro Universitário do Sul de Minas Universidade Federal de Lavras sheldonwilliamsilva@gmail.com

Pedro dos Santos Portugal Jr.

Centro Universitário do Sul de minaspedrorotaract@hotmail.com

Letícia Rodrigues da Fonseca Universidade Vale do Rio Verde leticia.rodrigues.vga@gmail.com

Sérgio Ricardo Magalhães Universidade Vale do Rio Verde sergio.magalhaes@unincor.edu.br

\section{O ESTUDO DE CASO NOS ESTUDOS ORGANIZACIONAIS: POTENCIAL E DESAFIOS}

\begin{abstract}
RESUMO
Os estudos de caso são investigações empíricas detalhadas sobre fenômenos complexos, com uma perspectiva que enfatiza a singularidade e o contexto do caso, para tanto, os pesquisadores utilizam de uma variedade de fontes de dados. Estes estudos, geralmente, combinam métodos diversos para coleta de dados, tais como: arquivos, entrevistas, questionários, observações. O objetivo deste ensaio é discutir o potencial e os desafios da utilização dos estudos de casos nas pesquisas organizacionais. Vários procedimentos são necessários para que se atinja a compreensão profunda da complexidade dos fenômenos e comportamentos sociais, entre esses procedimentos destacam-se: a utilização de múltiplas fontes de evidência, a triangulação dentro do método e entre métodos e teorização a partir de estudos de casos, ponto central deste ensaio teórico.
\end{abstract}

Palavras-chave: Estudo de caso. Estudos organizacionais. Métodos e Procedimentos. Teorização.

\section{THE CASE STUDY IN ORGANIZATIONAL STUDIES: POTENTIAL AND CHALLENGES}

\begin{abstract}
Case studies are detailed empirical investigations of complex phenomena with a perspective that emphasizes the uniqueness and context of the case, for which researchers use a variety of data sources. These studies generally combine diverse methods for data collection, such as: files, interviews, questionnaires, observations. The purpose of this article is to discuss the potential and challenges of using case studies in organizational research. Several procedures are necessary to achieve a deep understanding of the complexity of phenomena and social behaviors. Among these procedures, the following stand out: the use of multiple sources of evidence, triangulation within the method and between methods and theorization from case studies, central point of this theoretical essay.
\end{abstract}

Keywords: Case study. Organizational research. Methods and Procedures. Theorization.

Recebido em: 10/10/2017 - Aprovado em: 01/02/2018 - Disponibilizado em: 15/07/2018 


\section{INTRODUÇÃO}

Antes de ingressar no debate sobre o estudo de caso nos estudos organizacionais, cabem duas perguntas: "O que é um caso?" ou “O que são estudos de casos?” Essas questões podem ser respondidas sob diferentes perspectivas, não existindo um consenso sobre a sua definição no âmbito das ciências sociais. A discussão é profícua e merece atenção por parte dos pesquisadores, de modo que o caso ou estudo de caso possa ser empregado da maneira mais adequada ao contexto da pesquisa realizada e aos objetivos que a mesma se destina a alcançar.

Para alguns, o caso pode ser teórico ou empírico, ou ambos; pode ser um objeto ou um processo relativamente limitado; e pode ser genérico e universal ou específico (RAGIN, 1992). De maneira geral, o público-alvo das pesquisas das ciências sociais espera que os resultados dos estudos da área estejam fundamentados em provas empíricas, sendo a observação de muitos casos ou casos variados, uma base confiável para que os cientistas da área possam fundamentar os seus argumentos (RAGIN, 1992).

Os estudos de casos podem implicar a utilização de um único ou vários casos, assim como, vários níveis de análise (EISENHARDT, 1989). Os casos podem ser sistemas específicos, exclusivos e limitados, que podem ser definidos por indivíduos, por um grupo, por uma organização, por uma comunidade, por uma nação, por um episódio ou por um encontro, por um evento, por um período de tempo ou um processo sustentado ou espacial e temporal (RIDDER, HOON \& MCCANDLESS, 2009).
Os estudos de caso são investigações empíricas detalhadas sobre fenômenos complexos, com uma perspectiva que enfatiza a singularidade e o contexto do caso, para tanto, os pesquisadores utilizam de uma variedade de fontes de dados. Eles envolvem o inquérito concentrado sobre a complexidade dos casos, sendo uma ferramenta valiosa para fazer uma contribuição teórica na área de estratégia e na pesquisa em administração (RIDDER, HOON \& MCCANDLESS, 2009).

Não há uma definição precisa e exclusiva do que é um caso ou estudo de caso, porém a discussão sobre o assunto é importante para a evolução das pesquisas nas ciências sociais. Embora essa proposta de discussão já seja bastante antiga, observa-se ainda hoje na academia a aplicação equivocada do conceito estudo de caso e em alguns momentos uma dificuldade de se estabelecer uma definição adequada do que é um caso e como analisa-lo.

Diante desse contexto, o objetivo deste ensaio é discutir o potencial e os desafios da utilização dos estudos de casos nos estudos organizacionais. O ensaio está estruturado em cinco seções: após esta introdução, segue-se a segunda seção que discute os desafios e oportunidades da utilização dos estudos, a terceira seção que apresenta alguns dos procedimentos mais importantes para $\mathrm{o}$ desenvolvimento dos estudos de casos, assim como, os principais métodos de coleta e análise de dados, a quarta seção na qual se apresenta a teorização a partir de estudos de casos e por último a quinta seção onde são apresentadas as considerações finais. 


\section{DESAFIOS E OPORTUNIDADES DA UTILIZAÇÃO DE CASOS}

O estudo de caso pode ser feito por meio de qualquer evidência seja ela qualitativa ou quantitativa. A prova pode vir de dados primários (trabalho de campo, entrevistas, relatos verbais, observação) ou de dados secundários (documentos de arquivo), ou ainda da combinação destes se apresenta no decorrer deste ensaio.

O estudo de caso caracteriza-se por examinar: (a) um fenômeno contemporâneo em seu contexto de vida real, especialmente, quando (b) os limites entre o fenômeno e o contexto não são claramente evidentes (YIN, 1981). Portanto, é necessária a distinção entre os meios de prova, métodos de coleta de dados e estratégias de pesquisa para definição de estudos de caso.

Os estudos de casos, geralmente, combinam métodos diversos para coleta de dados, tais como: arquivos, entrevistas, questionários, observações. As provas podem ser qualitativas, quantitativas ou ambas (EISENHARDT, 1989). A utilização de várias provas pode contribuir para o aumento da confiabilidade do estudo de caso. Porém, a autora faz uma observação importante: a coleta de dados deve ser baseada no foco da pesquisa, sem esse foco de investigação bem definido, é provável que o pesquisador ficará sobrecarregado pelo volume de dados.

A análise dos estudos de caso mostra que a criação de oportunidades para a construção de uma contribuição teórica necessita da utilização de múltiplas lentes. Ao analisar um fenômeno com diversos olhares, novas percepções e entendimentos são gerados, o que não seria possível utilizando-se de uma única lente (RIDDER, HOON \& MCCANDLESS, 2009).

Há um amplo consenso entre os estudiosos de que os pontos fortes da pesquisa de estudos de caso residem na compreensão profunda da complexidade dos fenômenos e comportamentos sociais (RIDDER, HOON \& MCCANDLESS, 2009). Apesar da importância do estudo de caso, ainda há um predomínio de publicações nos principais periódicos de gestão, de artigos que empregam métodos estatísticos, com a utilização de múltiplos casos (MARIOTTO, ZANNI \& MORAES, 2014).

Uma das principais críticas sobre a utilização de estudos de casos é pela falta de utilização de critérios científicos padronizados. Essa crítica vem, principalmente, de estudiosos de orientação de uma "ciência normal", que reconhecem que estudos de casos podem ser utilizados, porém são adequados apenas nas fases preliminares da pesquisa, quando as variáveis ainda estão sendo exploradas (MARIOTTO, ZANNI \& MORAES, 2014).

\section{PRINCIPAIS MÉTODOS PARA COLETA E ANÁLISE DE DADOS}

Conforme discutido na seção anterior, a utilização de múltiplas fontes de evidências contribui para maior robustez dos resultados da pesquisa qualitativa de estudo de caso. Entre os métodos mais utilizados para a coleta de dados primários na pesquisa qualitativa destacam-se: (i) as entrevistas individuais e grupais; (ii) a etnografia. Nesse mesmo sentido, duas formas de 
análise dos dados merecem destaque no desenvolvimento de pesquisas qualitativas: (i) a análise de conteúdo; (ii) a análise do discurso. A seguir apresenta-se uma breve discussão sobre esses métodos de coleta e análise de dados em estudos de casos.

\subsection{Entrevistas individuais e grupais}

Os estudos de casos envolvem variadas formas de coletas de dados, o pesquisador pode trabalhar com dados de entrevistas, dados de arquivos, observações, livros históricos. Mas as entrevistas são frequentemente as fontes de dados mais utilizadas. As entrevistas são uma forma eficiente para coletar dados empíricos de forma detalhada. Para aumentar o nível de confiança da pesquisa e evitar possíveis vieses é importante aumentar o número de entrevistas no estudo de caso, entrevistar diferentes níveis hierárquicos, áreas geográficas, assim como, intervenientes de outras organizações e agentes externos (EISENHARDT \& GRAEBNER, 2007).

A utilização das entrevistas está ligada ao próprio contexto da pesquisa nas ciências sociais, que possui um pressuposto que o mundo social é "construído por pessoas em suas vidas cotidianas", nas ciências sociais a entrevista é uma técnica muito empregada (GASKELL, 2003, p. 65). Dessa forma, a utilização de entrevistas contribui para mapear e compreender essa realidade socialmente construída por meio da "compreensão das relações entre os atores sociais e sua situação" (p. 65).

A utilização de entrevistas, entretanto, requer alguns cuidados. Para Gaskell (2003), é necessário preparação e planejamento, que envolve a elaboração de um tópico guia, a seleção dos entrevistados, a definição de quantas entrevistas serão necessárias e a escolha metodológica: entrevistas grupais ou entrevistas individuais.

Um aspecto importante no desenvolvimento de entrevistas é a seleção dos entrevistados. Diferente da abordagem quantitativa, onde são empregados métodos estatísticos sobre a população, essa perspectiva não é aplicável na pesquisa qualitativa. De acordo com Gaskell (2003, p. 68), “a finalidade real da pesquisa qualitativa não é contar opiniões ou pessoas, mas ao contrário, explorar o espectro de opiniões, as diferentes representações sobre o assunto em questão".

Dessa forma, a seleção dos entrevistados deve ser realizada com "o objetivo de maximizar a oportunidade de compreender as diferentes posições tomadas pelos membros do meio social" (GASKELL, 2003, p. 68-69). Ainda de acordo com o autor, não existe um método específico para a seleção de entrevistados, o entrevistador deve usar a sua "imaginação social científica”.

Além da preocupação com a seleção dos entrevistados, existe também a preocupação com o número de entrevistas. Novamente, não existe uma resposta exata para essa questão, a definição do número de entrevista depende dos diferentes ambientes e contextos sociais pesquisados. Mas, uma ocorrência importante pode ajudar a definir o momento de cessar o desenvolvimento de novas entrevistas, quando temas comuns começam a aparecer e aos poucos o pesquisador percebe que novas informações não surgem, 
portanto, é um sinal que é o momento de parar (GASKELL, 2003), tal momento é denominado saturação teórica (EISENHARDT, 1989), quando novos casos ou novas entrevistas começam a repetir o que já foi coletado, não trazendo informações adicionais.

\subsection{Etnografia}

O método etnográfico tem sido elogiado pela sua utilidade na indução da teoria. A etnografia é um método de pesquisa desenvolvido especificamente para descrever fenômenos culturais. Considerando a etimologia da palavra, etnografia é a escrita sobre cultura, sendo esse método criado e amplamente utilizado nos estudos antropológicos (BRANNEN, 2011).

A etnografia consiste na imersão do pesquisador na rotina diária dos nativos. $\mathrm{O}$ pesquisador deve estar presente no momento em que ocorrem fatos importantes, pois os comentários e os sentimentos dos nativos vão aflorar e é preciso acompanhar tais manifestações (CAVEDON, 2014).

O rigor científico está presente na coleta e análise dos dados. O pesquisador deve estar atento aos objetivos da pesquisa, mas ao mesmo tempo, para melhor aproveitamento do método é possível realizar alterações no projeto, à medida que novos acontecimentos e fatos observados indicam novos caminhos. É preciso atentar para novos achados e rever postulados, evitando préconceitos (CAVEDON, 2014). O aspecto mais singular da etnografia em relação a outras metodologias de pesquisa é o próprio pesquisador. $\mathrm{O}$ pesquisador, como observador participante, desempenha papel fundamental na construção do estudo (BRANNEN, 2011).

O uso do método etnográfico nos estudos organizacionais requer atenção para algumas características da área. A primeira é que se trata de uma ciência aplicada, portanto, espera-se que as pesquisas tenham uma funcionalidade, ou seja, uma utilidade, uma aplicabilidade. Outro aspecto importante diz respeito à entrada em campo nas organizações. O primeiro contato, normalmente, é realizado com a cúpula da organização, a quem o etnógrafo precisa deixar bem claro os objetivos da pesquisa, assim como, as caraterísticas do método, que é "invasivo", "e isso pode trazer à tona significados que a administração da organização entende serem positivos como também significados que podem ser interpretados como negativos" (CAVEDON, 2014, p. 82)

\subsection{Análise de conteúdo}

A análise de conteúdo pode ser dividida em três etapas, conforme a seguir: (i) a préanálise; (ii) a exploração do material; (iii) o tratamento dos resultados, a inferência e a interpretação.

A primeira etapa da análise de conteúdo é denominada, por Bardin (2011) como préanálise. Nessa etapa duas ações são tomadas: (i) leitura flutuante, onde o pesquisador fará a leitura de documentos que estão sendo analisados e aos poucos essa leitura vai tomando forma e se tornando mais precisa; e (ii) a escolha dos documentos: os documentos são escolhidos e organizados, compondo o corpus da pesquisa. Nessa primeira fase, o pesquisador escolhe os 
documentos, formula as hipóteses e elabora indicadores que vão nortear a interpretação final.

Desenvolvida a primeira etapa de organização da análise, passa-se para a etapa seguinte que é conhecida como codificação. O processo de codificação diz respeito ao tratamento do material. Segundo Bardin (2011, p. 133), "tratar o material é codifica-lo". Na etapa da codificação o pesquisador deve levar em consideração duas unidades: (i) a unidade de registro e (ii) a unidade de contexto.

A terceira etapa é a categorização, que se trata de "uma operação de classificação dos elementos constitutivos de um conjunto por diferenciação e, em seguida, por reagrupamento segundo o gênero (analogia), com os critérios previamente definidos" (BARDIN, p. 141), em outras palavras, trata-se da definição de categorias, que podem ser adotadas considerando critérios: semântico (temas), sintático (verbos, adjetivos e pronomes), léxico (sentido e significado das palavras - antônimo ou sinônimo) e expressivo (variações na linguagem e na escrita). O principal objetivo dessa etapa é fornecer, por meio da condensação, uma representação simplificada dos dados brutos.

A quarta etapa apresentada por Bardin (2011) trata-se da inferência. Para a autora, a inferência é orientada pelos mecanismos constitutivos da comunicação: (i) a mensagem (significação e código) e o seu suporte ou canal; (ii) o emissor e o receptor. Esses polos de comunicação fazem parte da análise de conteúdo. Sendo que, o emissor é aquele que produz a mensagem, podendo ser um indivíduo ou um grupo. $\mathrm{O}$ receptor trata-se daquele que recebe a mensagem e estuda sobre a que ela se destina, podendo ser um indivíduo ou grupo. A mensagem trata-se do ponto de partida da análise, onde serão estudados o conteúdo, o significado, os significantes, o código e a significação. E o canal é mais como procedimento experimental do que para análise de conteúdo.

$\mathrm{Na}$ fase de interpretação dos dados, o pesquisador precisa retornar ao referencial teórico, procurando embasar as análises dando sentido à interpretação. Uma vez que, as interpretações pautadas em inferências buscam o que se esconde por trás dos significados das palavras para apresentarem, em profundidade, o discurso dos enunciados.

\subsection{Análise do discurso}

Existe uma variedade significativa de definições e enfoques no estudo de textos. Porém, ambas as perspectivas compartilham de duas conviç̧ões: (i) rejeitam que a linguagem seja simplesmente um meio neutro de refletir, ou descrever o mundo; (ii) assumem a importância central do discurso na construção da vida social (GILL, 2003). Apesar da diversidade de concepções sobre a análise de discurso, há quatro características-chaves que norteiam essas perspectivas: (i) uma postura crítica com respeito ao conhecimento dado, aceito sem discussão; (ii) o reconhecimento de que as maneiras como cada indivíduo compreende o mundo é histórica e culturalmente específica e relativa; (iii) a convicção de que o conhecimento é socialmente construído; e (iv) o compromisso de explorar as maneiras com os conhecimentos estão ligados a ações/práticas (GILL, 2003). 
Ainda segundo o mesmo autor, a análise do discurso possui quatro temas principais: (i) uma preocupação com o discurso em si mesmo; (ii) uma visão da linguagem como construtiva (criadora) e construída; (iii) uma ênfase no discurso como uma forma de ação; e (iv) uma convicção na organização retórica do discurso.

A análise do discurso trata-se de uma leitura cuidadosa, que caminha entre o texto e o contexto, para analisar o conteúdo, a organização e as funções do discurso (GILL, 2003).

"A análise de discurso não pode ser usada para tratar os mesmos tipos de questões como enfoques tradicionais. Ela sugere, ao invés, novas questões, ou maneiras, de reformar as antigas" (GILL, 2003, p. 245). A utilização da análise de conteúdo requer a utilização de perguntas em formatos bem diferentes das convencionais perguntas sociocientíficas.

\section{A TEORIZAÇÃO A PARTIR DE ESTUDOS DE CASO}

Os estudos de casos podem ter finalidades distintas. Eles podem ser descritivos, podem testar uma teoria ou ainda gerar uma nova teoria. Eisenhardt (1989) destaca a importância do estudo de caso utilizado para gerar uma nova teoria. Para a autora, a teoria resultante dos estudos de casos é muitas vezes nova, testável e empiricamente válida, para tanto, esse tipo de pesquisa precisa ser estruturado e ter uma fundamentação convincente, sendo esses os critérios-chaves para a avaliação desse tipo de pesquisa.
Uma crítica comum aos estudos de caso é entender que não se pode generalizar os resultados de uma pesquisa a partir de um caso individual, excluindo assim a capacidade desse estudo de realizar uma contribuição científica. Para Ridder, Hoon e McCandless (2009), os estudos de caso são apropriados para mostrar violações de uma teoria e entender o motivo pelo qual os resultados diferem do que era esperado, produzindo resultados inesperados e ricos em oportunidades para criar uma nova teoria. Além disso, os estudos de caso podem ser fontes ricas de pesquisa para a ampliação de teorias já existentes.

A construção de teoria a partir de estudos de caso trata-se de uma estratégia de investigação que envolve o uso de um ou mais casos para criar construtos, proposições e/ou teoria, baseado em evidências empíricas. Um ponto central para construir uma teoria a partir de estudos de caso é a lógica de replicação (EISENHARDT \& GRAEBNER, 2007).

O desenvolvimento de uma teoria a partir de uma investigação empírica começa com forte embasamento na literatura do tema foco da pesquisa, identificando uma lacuna e propondo perguntas de pesquisa que abordem esse foco, além disso, os pesquisadores devem justificar de forma clara e coerente a escolha do método de pesquisa a partir do problema abordado (EISENHARDT \& GRAEBNER, 2007).

Oportunidades para fazer uma contribuição teórica são ativadas à medida que os conceitos teóricos e suas relações são suscetíveis de serem validadas em diferentes contextos. Ao transferir a abordagem teórica em um novo contexto empírico, o estudo de caso 
pode dar uma contribuição teórica (RIDDER, HOON \& MCCANDLESS, 2009).

O pesquisador deve ser capaz de deixar evidenciado na justificativa e problematização do estudo a razão pela qual não há uma teoria existente que ofereça uma resposta viável. Via de regra, esse método de pesquisa é justificado pela importância do fenômeno e pela falta de alterativas de teoria, que serão construídas ou alargadas a partir da evidência empírica, na capacidade que os dados qualitativos têm de oferecer insights em complexos processos sociais que os dados quantitativos não podem facilmente revelar (EISENHARDT \& GRAEBNER, 2007).

Outro aspecto importante nos estudos de casos trata-se da seleção dos casos. Um possível questionamento que pode surgir ao se apreciar o resultado de um estudo de caso é o seguinte: como é possível generalizar uma teoria se os casos não são representativos? Uma resposta adequada para essa pergunta é demonstrar que o propósito do estudo é desenvolver teoria e não a testar. Sendo assim, a escolha dos casos não é realizada por amostragem, os casos devem ser selecionados pela sua capacidade particular de contribuir para a criação ou alargamento de uma teoria, em outras palavras, os casos são escolhidos porque eles são reveladores, permitem a exploração de um fenômeno raro (EISENHARDT \& GRAEBNER, 2007).

$\mathrm{O}$ pesquisador deve selecionar os casos ou o caso propositalmente com a finalidade de explorar um problema em profundidade e em grande detalhe. A escolha do caso é uma das etapas mais importantes do processo de pesquisa do estudo de caso. Diferente da amostragem estatística, a seleção dos casos aqui é tratada como uma escolha do pesquisador, de acordo com a capacidade que o caso tem de contribuir para o fenômeno estudado (RIDDER, HOON \& MCCANDLESS, 2009).

Casos únicos, surpreendentemente, podem gerar teorias muito mais complexas do que casos múltiplos, isso acontece porque em estudos de casos únicos, o pesquisador pode aplicar sua teoria exatamente aos muitos detalhes de um caso particular. Em contrapartida, em estudos de casos múltiplos pesquisadores conservam apenas os relacionamentos que são replicados entre a maioria ou entre todos os casos (EISENHARDT \& GRAEBNER, 2007).

A teorização a partir de estudos de casos pode ser legitimada a partir do cuidado com a justificação do estudo, da seleção adequada dos casos, da diversificação das entrevistas para reduzir os possíveis vieses da pesquisa, da apresentação rica de provas em tabelas e apêndices, e da clareza na argumentação teórica. O resultado disso é uma teorização a partir de estudos de caso rica e consistente (EISENHARDT \& GRAEBNER, 2007).

\section{CONSIDERAÇÕES FINAIS}

Os estudos de casos têm ganhado espaço nas pesquisas com a abordagem de variados temas (EISENHARDT \& GRAEBNER, 2007). Esse tipo de pesquisa tem um potencial significativo de contribuição para os estudos organizacionais, especialmente, para a teoria da estratégia e da gestão (RIDDER, HOON \& MCCANDLESS, 2009). A realização de estudos de casos nas ciências sociais aplicadas pode 
contribuir para a construção e evolução da teoria, desde que bem planejados, arquitetados e desenvolvidos. A utilização de múltiplas fontes de evidências, a exploração profunda do(s) caso(s), assim como, a triangulação permitem a teorização a partir dos estudos qualitativos. Nesse sentido, o objetivo deste ensaio foi discutir o potencial e os desafios da utilização nos estudos de casos nos estudos organizacionais.

O estudo de caso(s) trata-se de uma estratégia de pesquisa que incide sobre o entendimento da dinâmica dentro da configuração de um determinado fenômeno. Normalmente, desenvolvido a partir de um ou mais casos que são selecionados pelas suas características particulares e pela sua capacidade de contribuir para o desenvolvimento e/ou ampliação da(s) teoria(s) existente(s).

As principais oportunidades oriundas das pesquisas do tipo estudo de caso residem na flexibilidade e diversidade em termos de métodos de coleta e análise dados. $\mathrm{O}$ pesquisador pode e deve, na medida do possível, utilizar múltiplas fontes de evidências, com o intuito de aplicar a triangulação, dentro do próprio método e entre métodos, utilizando assim de dados primários e secundários, qualitativos e quantitativos. Tornando, portanto, os resultados das pesquisas mais robustos e confiáveis.

A principal oportunidade defendida neste ensaio é a teorização a partir dos estudos de casos. Embora notada resistência por muitos pesquisadores, importantes teóricos dos estudos organizacionais, como Eisenhardt (1989), Yin (2005) Eisenhardt e Graebner (2007), Ridder, Hoon e McCandless (2009), defendem que é possível contribuir para o desenvolvimento e evolução da teoria a partir da utilização de estudos de casos, entretanto, para que esse objetivo seja alcançado o pesquisador deve se preocupar com o rigor e profundidade da pesquisa, buscando exaurir as fontes de evidências, aplicando o conceito da saturação teórica. Finalmente, a triangulação dos dados coletados com a teoria existente pode ajudar a reduzir e/ou preencher lacunas, assim como, corroborar ou até mesmo refutar teorias existentes.

Mesmo diante de tantas oportunidades muitas são as críticas aos estudos de casos. Matthew Miles (1979) fez duras críticas à pesquisa qualitativa nos estudos organizacionais, em seu artigo "Qualitative Data as an Attractive Nuisance: The Problem of Analysis", ao afirmar que tais pesquisas não passam de "contações" de histórias, apontando erros graves da pesquisa organizacional e deixando poucas esperanças para o futuro da área. Trazendo essa reflexão para o contexto atual, mais de trinta anos se passaram e será que a crítica Miles continua a ressoar no campo das pesquisas sociais? Qual a validade e contribuições dos estudos de casos atuais? São todos estruturados e organizados de forma clara ou continuam sendo narrativas longas que não seguem uma estrutura previsível, conforme apontado por Yin (1981)? Certo é que o estudo de caso é uma importante fonte para o desenvolvimento e evolução das ciências sociais, especialmente, dos estudos organizacionais, porém, mesmo diante dessa significância os estudos de casos ainda demandam de um maior rigor por parte de muitos pesquisadores. 


\section{REFERÊNCIAS}

BARDIN, L. Laurence. Organização da Análise; A codificação; A categorização; A inferência. In.: BARDIN, L. Laurence. Análise de conteúdo. São Paulo: Edições 70, p. 125-171, 2011.

BRANNEN, M.Y. Using multiple case studies to generalize from ethnographic research. In.: Piekkari, R. \& Welch, C. (org.). Rethinking the Case Study in International Business and Management Research. Northampton: Edward Elgar Publishing, 2011.

CAVEDON, N.R. Método etnográfico: da etnografia clássica às pesquisas contemporâneas. In.: SOUZA, E.M. (org.). Metodologias e analíticas qualitativas em pesquisa organizacional: uma abordagem teóricoconceitual. Vitória : EDUFES, 2014.

EISENHARDT, K.M. \& GRAEBNER, M.E. Theory Building From Cases: Opportunities And Challenges. Academy of Management. n. 1, v. 50, p. 25-32, 2007.

EISENHARDT, K.M. Building Theories from Case Study Research. Academy Of Management Review, v.14, n.4, 532-550, 1989.

GASKELL, G. Entrevistas individuais e grupais. In: Gaskell, G . Pesquisa qualitativa com texto, imagem e som: um manual prático. (3a ed), Petrópolis: Vozes, 2003.

GILL, R. Análise de discurso. In.: BAUER, M.W. \& GASKELL, G. (eds.), Pesquisa Qualitativa com Texto, Imagem e Som: Um manual prático. Petropólis: Editora Vozes, p. 244-270, 2003.

JICK, T.D. Mixing Qualitative and Quantitative Methods: Triangulation in Action. Administrative Science Quarterly, 24, p.602-369, 1979.

MARIOTTO, F.L., ZANNI, P.P. \& MORAES, G.H.S.M. What is the of a single-case study in management research? Revista de Administração de Empresas, n.54, v.4, p.358-369, 2014.

MATTHEW, B.M. Qualitative Data as an Attractive Nuisance: The Problem of Analysis. Administrative Science Quarterly. Qualitative Methodology. n. 4. v. 24, p. 590-601, 1979.

RAGIN, C.C. What's a case? Exploring the Foundation of Social Inquiry, Cambridge University Press, 1992.

Ridder, H. \& Hoon, C. \& McCandless, A. The theoretical contribution of case study research the fiel of strategy and management. Research Methodology in Strategy and Management, v. 5, p. 137-175, 2009.
YIN, R.K. The Case Study Crisis: Some Answers. Administrative Science Quarterly, n.26, n.1, p.58-65, 1981.

YIN, R.K. Conduzindo estudos de casos: coleta de evidências. In: Yin, R. K . Estudo de caso: planejamento e métodos. Porto Alegre: Bookeman,2005.

Reginaldo da Silva Souza
Professor UninCor, Doutorando em Administração
pela PUC-Minas, Mestre em Administração pela
FPL, Especialista em Gestão Empresarial e Graduado
em Administração pela FACECA.

\section{Sheldon William Silva}

Professor UNIS-MG, Doutorando em Administração pela UFLA, Mestre em Administração pela FPL, Especialista em Gestão Empresarial e graduado em Administração com Habilitação em Comércio Exterior pela Universidade do Estado de Minas Gerais.

\section{Pedro dos Santos Portugal Jr.}

Professor UNIS-MG, Doutor e Mestre em Desenvolvimento Econômico pelo Instituto de Economia da Unicamp, Especialista em Gestão de Negócios e graduado em Ciências Econômicas pela FACECA.

\begin{tabular}{l}
\hline Letícia Rodrigues da Fonseca \\
Professora do curso de Mestrado em Sustentabilidade \\
em Recursos Hídricos da UninCor - Universidade \\
Vale do Rio Verde. Doutora em Administração pela \\
Universidade Presbiteriana de Mackenzie. \\
\hline
\end{tabular}

Sérgio Ricardo Magalhães
Professor do curso de Mestrado em Sustentabilidade
em Recursos Hídricos da UninCor - Universidade
Vale do Rio Verde. Doutor em Engenharia
Biomédica pelo IP\&D - SJC.

